\title{
LA VIDA COTIDIANA DE LAS ORGANIZACIONES POPULARES COMO ESPACIO FORMATIVO
}

\section{Resumen}

El artículo sintetiza una parte de los resultados de la investigación Saberes y prácticas pedagógicas en organizaciones populares, cofinanciada por la DGP-CIUP y realizada por investigadores de la línea Memoria, identidad $y$ actores sociales. En particular, se centra en la vida cotidiana de las asociaciones populares como un espacio valorado por sus integrantes como formativo. A la luz de los aportes de algunos estudiosos de la vida cotidiana y partir de la reconstrucción histórica de la experiencia educativa de las organizaciones y de un trabajo de campo de carácter etnográfico, el texto analiza los espacios, momentos, contenidos y formas de aprendizaje en la cotidianidad de las organizaciones. Finalmente, concluye que la confluencia entre las prácticas educativas formales y la experiencia dentro de la vida cotidiana de las organizaciones, es la que garantiza un propósito central de éstas: la formación de sus integrantes como sujetos sociales.

Palabras clave: Organización popular,vida cotidiana, formación. necesidades, sujeto social.

\section{Abstract}

This article synthesizes a part of the results of the investigation "Knowledge and practical pedagogic in popular organizations" cofinanced by the DGP -CIUP and carried out by investigators of the Line Memory, identity and social actors. In particular, it is centered in the daily life of the popular associations as a space valued by their members as formative. By the light of the contributions of some specialists of the daily life and to leave of the historical reconstruction of the educational experience of the organizations and of a field work; the text, too analyzes the spaces, moments, contents and learning forms in the daily life of the organizations. Finally, it concludes that the confluence between the formal educational practices and the experience inside the daily life of the organizations is what guarantees a central purpose of these: the formation of their members as social actor.

Key words: Popular organization, daily life, formation, necessities, social actor.

\footnotetext{
"Profesor Universidad Pedagógica Nacional, Bogotá. E-mail: atorresc@multiphone.net.co
} Texto recibido el 12 de abril de 2004 y aprobado el 18 de mayo de 2004. 


\section{Presentación}

Es un hecho reconocido que las organizaciones populares promueven intencionalmente acciones de formación y capacitación de sus miembros y de los participantes de sus proyectos; en tal sentido, realizan eventos y desarrollan procesos explícitamente educativos, como charlas, talleres, cursos y escuelas de organización, con unos contenidos y unas metodologías coherentes con sus organización ideológicas y pedagógicas. En la mayoría de los casos, también crean espacios destinados a la reflexión pedagógica sobre dichas acciones educativas.

Junto a este "currículo manifiesto" de las organizaciones populares, la investigación ${ }^{1}$ nos permitió reconocer que existen otros espacios y momentos de la vida diaria de las mismas, a los que sus participantes atribuyen una gran incidencia en su transformación personal, en la transformación de sus modos previos de ver, valorar y actuar. En efecto, tanto en la reconstrucción histórica de sus prácticas pedagógicas como en el trabajo de campo, nos llamó la atención la frecuente valoración que hacen los participantes de su paso por la organización como experiencia educativa, como lo testimonia una educadora de la EPI de Villa Nidia: "Yo quiero hablar de mi experiencia, y yo he estado como en varios procesos aquí en la organización, y todos me han formado en varios momentos".

Por otro lado, para sus dirigentes, las organizaciones son asumidas como "proyectos educativo culturales", en donde todas sus actividades y espacios estarían orientados a formar a sus participantes como sujetos de dichos proyectos; por

\footnotetext{
' Nos referimos al Proyecto Saberes y prácticas pedagógicas en tres organizaciones populares, realizado por la línea de investigación Memoria, identidad y actores sociales, del Grupo de investigación Sujetos y Nuevas Narrativas en Ciencias Sociales, reconocido por Colciencias, y apoyado por el DG - CIUP, UPN.
}

ejemplo, para el Centro de Promoción y Cultura, "en el CPC todo es educativo... Io educativo atraviesa todas las prácticas del CPC desde sus dos líneas de acción: evangelización y transformación". Es decir, que además de las prácticas intencionalmente educativas, la propia dinámica cotidiana de las organizativas es considerada como formativa.

Dichas apreciaciones coinciden con lo encontrado en estudios similares, según los cuales se reconoce que las organizaciones y los movimientos sociales son decisivos en los cambios de representaciones, valores y formas de relación interpersonal de sus miembros, así como en la redefinición de su sistema de necesidades, de sus referentes de identidad y de sus prácticas sociales, más allá del ámbito asociativo y de los movimientos ${ }^{2}$.

Este reconocimiento del importante papel educativo que juega la vida cotidiana de las organizaciones, no sólo como contenido de sus prácticas pedagógicas ${ }^{3}$, sino como espacio de saber y aprendizaje, nos condujo a asumirla como una de las problemáticas a profundizar durante la fase interpretativa de la investigación. Sin embargo, un problema

${ }^{2}$ Ver al respecto: Salete, Roseli, "Los movimientos sociales y la formación de una nueva pedagogia", en El arco iris, Santiago, CEAAL, 1988; Palma, Diego, La construcción de Prometeo. La educación para la democracia latinoamericana. CEAAL-TAREA, Santiago 1993; Torres, Alfonso, "Educación y pedagogia en las experiencias organizativas populares", en La Piragua \# 11, CEAAL 1995; Martinic, Sergio, La construcción dialógica de saberes en contextos de educación popular, CEAAL, Santa Cruz de la Sierra, 1996; Sabucedo, J. M. y otros, "Los movimientos sociales: discurso y acción política", en Revista Universidad de Gualadajara, Número 11, verano de 1998.

${ }^{3}$ En las tres organizaciones estudiadas, 10 cotidiano aparece como contenido o momento didáctico de sus propuestas; así, por ejemplo, en las actividades formativas de FASOL, "se parte de las vivencias cotidianas de las mujeres", en la EPI, las Unidades pedagógicas Integradas, "parten de la realidad de los niños", y en Kerigma, "la vida de cada día, es la materia prima para las pues ta en escena". que encontramos para abordarlo es, que a diferencia de las prácticas educativas intencionales, los contenidos, espacios y formas de aprendizaje en la vida cotidiana, no son explícitas; en ella se ponen en juego representaciones, creencias, roles y formas de relación y de acción incorporadas por sus protagonistas como habitus 4 .

Por ello, para definir aquellos momentos, espacios, situaciones de la vida cotidiana en las organizaciones populares que potencialmente puedan adquirir la categoría de formativas, recurrimos a una lectura, "entre líneas", de los testimonios recogidos en la fase de reconstrucción histórica y de los registros etnográficos. Además, bajo el supuesto de que los agentes sociales pueden hacer reflexivas sus rutinas y prácticas cotidianas (Giddens:1995), propiciamos eventos de conversación con algunos de sus integrantes, en torno a lo que ellos consideraban como formativo dentro de la vida corriente de las organizaciones.

A partir de estas consideraciones, nos planteamos las siguientes preguntas, para dar cuenta de la potencialidad educativa de la cotidianidad de las organizaciones populares: ¿Cuáles momentos de la vida de las organizaciones son potencialmente formativos para sus integrantes? ¿Cuáles son los contenidos que se aprenden en la cotidianidad de la organización? ¿Cómo se aprehenden dichos saberes? ¿Cómo confluyen en la formación, otros aprendizajes provenientes otros ámbitos? ¿Cómo se procesan desde la vida cotidiana de las organizaciones las necesidades de los participantes en su formación?

${ }^{4}$ Asumimos este concepto central en la obra de Pierre Bourdieu, como "el sistema de disposiciones adquiridas, permanentes y transferibles que permiten actuar, percibir, sentir y pensar de una cierta manera... el habitus nos permite orientarnos en un espacio social sin necesidad de reflexión". En Téllez Gustavo, Pierre Bourdieu. Conceptos básicos y construcción socioeducativa. Bogotá, Universidad Pedagógica Nacional, 2002 


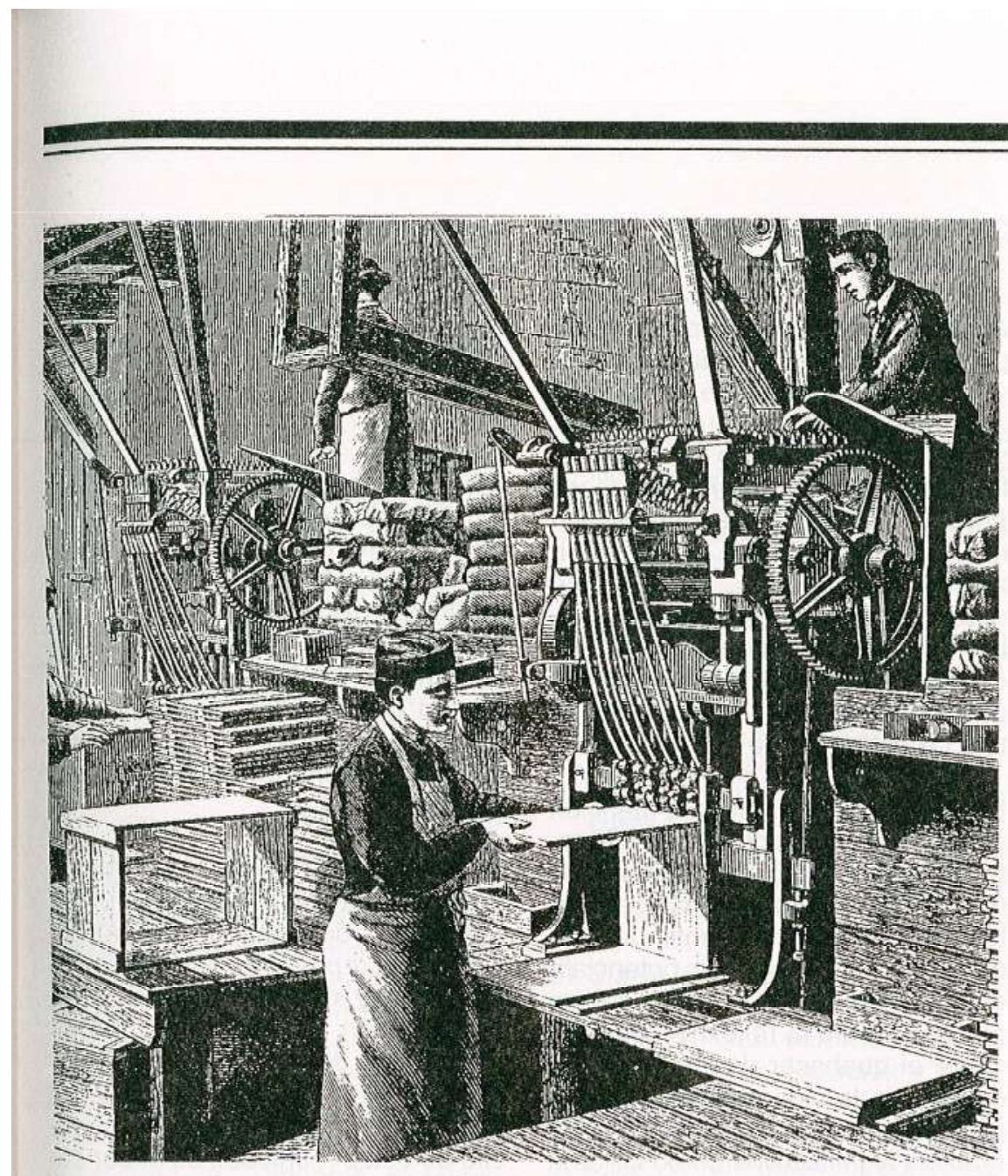

Este artículo sintetiza las conclusiones del proyecto "Saberes y prácticas pedagógicas en organizaciones populares"5, referidos a la vida cotidiana de las organizaciones como espacio formativo; están basadas en un diálogo entre la información obtenida, los referentes conceptuales a los que acudimos para interpretarla y el aporte de los demás integrantes del Proyecto de investigación. En primer lugar, sintetizamos nuestro posicionamiento conceptual frente a la categoría de vida cotidiana, para luego centrar la atención en los momentos, los contenidos y las formas de aprendizaje cotidiano en las organizaciones; finalmente, nos referiremos a la vida de las or-

${ }^{5}$ Las otras dos partes del informe, referidas a las prácticas educativas explicitas de las organizaciones, y a la formación como saber pedagógico de las organizaciones, serán publicadas posteriormente. ganizaciones como espacio de resignificación de necesidades y de aprendizajes provenientes de otros ámbitos en la perspectiva de formación de sus miembros como sujetos.

\section{La vida cotidiana como problema conceptual}

Antes de definir aquellos momentos, espacios, situaciones de la vida cotidiana en las organizaciones populares que potencialmente puedan adquirir la categoría de formativas, hemos considerado necesario, en primer lugar, explicitar desde dónde y cómo estamos entendiendo el concepto de vida cotidiana. Para ello, hemos acudido a algunos autores provenientes de tradiciones de pensamiento como la fenomenológica (Schutz, Berger y Luckman) y el marxismo (Héller y Kosik), así como a autores contemporáneos, como De Certeau, Maffesoli y Reguillo). A continuación, sintetizaremos, algunas ideas claves que nos sirvieron para construir el modelo de análisis e interpretar la problemática señalada.

En primer lugar asumimos que lo cotidiano es el conjunto de eventos que son conocidos y convividos como tales por los sujetos. Para Alfred Schutz (1993), la vida cotidiana es una esfera de la realidad en la que el individuo actúa de manera natural, la presupone desde su sentido común; es aquella parte de la vida que está dada y de la cual no cabe duda alguna; en ella se actúa rutinariamente sin prevención. En resumen, Schutz especifica cinco características de la vida cotidiana o mundo de la vida:

- Es presupuesta, su existencia nos antecede, es real y desde su sentido común el individuo la reconoce.

- Es intersubjetiva, se da por medio de una construcción recíproca entre los individuos.

- El individuo se encuentra en actitud natural frente a ella.

- En el mundo de la vida el individuo crea tipificaciones a partir de sus experiencias, valores, personas, las que son constituidas a través de la creación colectiva.

- En ella, se construye un acervo de conocimiento, el cual se refiere a los contenidos del mundo de la vida social que están referenciados dentro de un límite.

Para Karel Kosik (1979), la-vida cotidiana es, ante todo, la organización, día tras día, de la vida individual y colectiva de los hombres; la cotidianidad es la división del tiempo y el ritmo en que se desenvuelve la vida de cada cual y de cada grupo. Agnes Heller (1980), por su parte, considera que todos los ámbitos de la vida social tienen su propia cotidianidad, y por tanto ésta no hay que equipararla a la vida privada. La vida cotidiana es el espacio donde se dinamiza la reproducción 
social, a manera de socialización de las estructuras establecidas, las que finalmente son practicadas en el diario vivir de los individuos y en este proceso son interiorizadas.

Para Maffesoli (1992), la vida cotidiana no es solamente un contenido, sino es una perspectiva desde la cual se pueden abordar diferentes esferas sociales, es el plano de la socialidad, es decir, el de las relaciones básicas, cara a cara, donde no sólo se expresa el orden social, sino también se evidencian sus intersticios donde surge lo nuevo. En este mismo sentido, Michel de Certeau (1996) reivindica la vida cotidiana como el espacio donde se generan estilos de intercambio sociales, estilos de invenciones técnicas y un estilo de resistencia social; de este modo, la cotidianidad, más que reproducción y consumo, es producción de relaciones, de modos de hacer y de alternativas.

Este reconocimiento de la vida cotidiana no sólo como instituida sino como instituyente, la sintetiza Rosana Reguillo en los siguientes términos:"La vida cotidiana se constituye en un lugar estratégico para pensar la sociedad en su compleja pluralidad de símbolos y de interacciones ya que se trata del espacio donde se encuentran las prácticas y las estructuras, del escenario de la reproducción y simultáneamente de la innovación social" (Reguillo: 2000: 46).

En lo que respecta a esta investigación, consideramos que la vida cotidiana de las organizaciones constituye un espacio privilegiado para analizar en qué medida los nuevos sentidos de construcción de realidad que intencionalmente impulsan, se manifiestan en su quehacer diario, cómo se ve afectado por otros ámbitos y prácticas sociales de sus miembros y de qué manera en las organizaciones se están generando nuevos modos de interpretar la realidad y de "estar juntos", así como nuevas formas de acción social.

\section{Espacios y momentos formativos}

Además de ser valorada como espacio apreciado de encuentro y apoyo, la vida cotidiana de las organizaciones es vista por sus miembros y participantes como un espacio formativo, en "el que aprenden a toda hora". Si embargo, reconocer, sin más, que la vida diaria de la organización es formativa, no nos dice mucho; es necesario precisar cuáles espacios y tiempos son especialmente valorados por sus participantes como tales, en función del tipo de saberes que se generan.

Ello nos permitió centrar la atención en algunos espacios potencialmente formativos, por ser éstos donde más operan las transformaciones en los modos de ver, de sentir, de relacionarse y de hacer. La potencialidad de estos espacios radica en que allí se propicia la reflexión colectiva sobre el quehacer de las organizaciones, de la vida cotidiana de sus integrantes, asi como del devenir de ámbitos significativos como la familia, el barrio, el país; allí se expresan, se confrontan, reelaboran e interiorizan las maneras de entender, valorar y actuar en dichos ámbitos.

Entre estos espacios cotidianos formativos en las organizaciones, por ahora, diferenciamos entre unos más formales y otros más informales. En el primer caso, estarían todas aquellas instancias y espacios permanentes, creados por las propias organizaciones para planear, hacer seguimiento y evaluación de los proyectos y acciones que desarrollan; en segundo lugar, los momentos de celebración, de conmemoración y movilización; finalmente, los espacios informales asociados al quehacer rutinario de las organizaciones.

\section{Las reuniones}

En el caso de FASOL ${ }^{6}$, éstos son evidentes, dado que cada proyecto e instancia de coordinación realiza reuniones periódicas, las cuales también son vistas como ocasión privilegiada para hacer "acompañamiento" a las personas en formación. En primer lugar, están las reuniones periódicas en las cuales se planean las acciones a realizar y para evaluar, así como para ir construyendo el sentido mismo del proyecto; en segundo lugar, están las reuniones del equipo coordinador de FASOL, integrado por las 5 mujeres que poseen vínculos y responsabilidades permanentes dentro de la organización; este espacio se ha constituido en un espacio de reflexión grupal y de acercamiento entre las mujeres integrantes del equipo coordinador, "las duras de FASOL".

El Equipo coordinador de FASOL también participa en las reuniones mensuales del Equipo general del CPC; éste representa en espacio de encuentro y sentido de cuerpo de la organización. Se realiza los primeros viernes de mes y participan todos los miembros del Equipo del CPC (unas 35 personas); el propósito de estas reuniones es hacer seguimiento a las metas y tareas comunes, realizar celebraciones y abordar temas de formación sociopolítica y de coyuntura, como la Guerra en Irán y el Referendo.

En la Escuela Popular Infantil (EPI) del barrio Villa Nidia, son consideradas formativas las reuniones de trabajo de las educadoras, en particular, la Comisión EPI, las evaluaciones anuales y las asambleas que se realizaban con otras organizaciones de la "Coordinadora de Organizaciones de defensa de los derechos de los niños y las niñas":

La comisión EPI, ha sido un espacio que ha generado muchísimas inquietudes, pero también muchas respuestas, que ha generado errores, pero también a la vez cambios y

${ }^{6}$ Familias Solidarias, es un colectivo de mujeres que, apoyadas por el Centro de Promoción y Cultura (CPC), realizan actividades encaminadas a mejorar su calidad de vida, a organizarse y a afirmar su identidad como mujeres populares. 
trasformaciones, y se han ido generando. EPI: Entonces, un espacio ha sido ese, y otro han sido las diferentes asambleas a nivel de la coordinadora, le han dado a uno como muchos recursos para que uno se cualificara (Tertulia EPI).

En la Fundación Teatral Kerigma, no existen unos espacios de reunión fijos; sólo en momentos de crisis o cuando se presentan problemas coyunturales, se realizan reuniones para discutir, analizar y construir los criterios y acciones para superar dichas situaciones adversas.

\section{Celebraciones y movilizaciones}

Otro espacio considerado como formativo es la preparación y realización de eventos celebrativos, conmemorativos de expresión artística, los cuales son muy significativos en la vida e historia de las organizaciones, por su proyección local y nacional; es el caso de las celebraciones navideñas y del Carnaval por la Vida, para el CPC y de la Feria Cultural Hijos del Cerro, para la EPI; asi mismo, la Comparsa, la Muestra de Arte Popular y la participación en el Festival Iberoamericano de Teatro, son para Kerigma, ocasiones de cualificación de sus miembros.

Dichos eventos culturales, aunque son coyunturales, requieren un largo proceso de preparación, que implica establecimiento de acuerdos, discusión permanente, asunción de nuevas tareas y, por ende, formación y capacitación individual y colectiva, tal como lo recuerda Marisol en su historia de vida: "En esa Navidad se organizaron cursos para niños, entonces en esa organización de cursos para niños; por ejemplo, ellos empezaban bueno que quienes queríamos hacer actividades de cómo enseñar a hacer un pesebre; pero alrededor del pesebre, cómo trabajar valores con los niños".

Otro tipo de acontecimiento que requiere construcción de acuerdos, preparación y capacidad de nego- ciación, es la participación en jornadas de movilización, como fue el caso de las marchas y tomas del ICBF, por parte de la Coordinadora de Organizaciones, de la cual forma parte la EPI Villa Nidia:

eso fue a través de un lucha, y es una lucha política; sí, porque estábamos reclamando los derechos que uno diría que si no hubiera participado... no hubiesen habido esas inquietudes, pues no estaríamos de pronto en este cuento. Sí, fue un lucha dura, el hecho de ir y dormir allá una noche en las oficinas del ICBF, tomarnos las cocinas y hacer todas esas cosas, tumbar las rejas, meternos allá a las malas, aguantar bolillo y toda esa cuestión, eso no fue gratuito. ... Esas son cosas que se le van como metiendo a uno, y uno dice, es que lo que pasa es que hay que luchar para conseguir las cosas.

\section{El diario caminar}

Finalmente, son valorados como formativos, otros espacios y tiempos informales, como son los encuentros previos y posteriores a los eventos de formación, la conversación entre compañeros a lo largo de las rutinas diarias y los momentos casuales de celebración (un cumpleaños, la boda de una participante, un triunfo colectivo o individual); en la tertulia con las señoras de FASOL, expresaron que aprendían unas de otras:

En el diario caminar con las otras compañeras, en el chiste, en el cuento, en las relaciones mismas que establecemos entre nosotras cuando estamos haciendo las artesanías o el pan...

En el poder compartir en el núcleo un cafecito y una torta, en la realización de un baby shower a alguna compañera que tuvo un hijo..

Hemos aprendido a vivir y a sufrir con otras, compartiendo nuestros problemas.

A mí me da alegría encontrarme con ellas, no más, el hecho de repartir los lapicitos, para mí es muy importante...

Algo similar sucede en Kerigma, que se asume no sólo como organi- zación, sino también como "una posibilidad de interacción con los otros, con los amigos, aquellos con los que se han construido sueños posibles, se han enfrentado dificultades; con aquellos que están ahí para hablar, para reír al calor de un tinto, un cigarrillo y por qué no, un brandy", tal como lo expresan el siguiente testimonio: "Un día normal de Kerigma es llegar, tomar tinto, hablar con el Toño, hablar de lo sucedido o de lo que sucedió la noche anterior, hablar de los sucesos de pronto nacionales".

\section{Los contenidos del aprendizaje cotidiano}

Entendemos por contenidos de la formación, el conjunto de saberes (cognitivos, valorativos, actitudinales, prácticos) que los sujetos adquieren en una experiencia educativa y que puede usar autónomamente para asumir nuevas situaciones. A través de las acciones intencionalmente educativas, promovidas desde las organizaciones, enfatizan contenidos cognoscitivos y valorativos (el funcionamiento del Estado y de los derechos ciudadanos, conciencia crítica, solidaridad y compromiso), así como aquellos que desarrollan capacidades propias de los campos de acción y roles de sus integrantes:

De todas maneras, a mi me gustó mucho porque uno crea conciencia politica, el gobierno en ese momento, lo que significa un Estado, el gobierno que llega, qué hace, cómo lo hace, por qué dice que va a hacer y no hace; o sea, todo esto, realmente yo lo aprendí aquí. Y en eso tampoco me las puedo dar de dura... pero sí siento que tengo cierta conciencia, sentido político (Tertulia EPI).

Por otro lado, el ámbito de los espacios y momentos cotidianos considerados como formativos, también tiene sus propios contenidos; éstos se refieren, tanto a las formas de comprender(se) y valorar(se), como a los modos de actuar y relacionarse. En fin, el currículo cotidiano de 
las organizaciones lleva a que la gente, "redefina sus referentes identitarios y resignifique su vida cotidiana" (Maguenzo:1981: 181).

En el plano de los cambios cognitivos, en primer lugar, la experiencia cotidiana dentro de la organización, posibilita en sus participantes la adquisición de nueva información y ampliación de sus nociones sociales. Los participantes de las organizaciones señalan que conocen mejor la situación del barrio y del país, el funcionamiento de la política y sus derechos; también, que adquirieron conocimientos sobre el campo de acción en el que están (pedagogía, salud, teatro, enseñanza de la lectura y la escritura, etc.)

Pero más que información y conocimientos específicos, los partícipes de una experiencia asociativa amplían sus estrategias de comprensión de la realidad social, sus esquemas interpretativos (Berger y Luckmann:2003); dichos cambios de comprensión involucran simultáneamente, estructuras intelectuales, valorativas y prácticas; un ejemplo, es el reconocimiento, por parte de las educadoras de la EPI, del carácter político de la problemática infantil y de su labor educativa:

Algo que sí me hace pertenecer a esta organización: es el sentido político del trabajo con los niños. Entonces es trasformar pensamientos, maneras de ser; yo creo que es una de las partes más importantes que se crea acá en la organización; el trabajo que se realiza con los niños tiene también un sentido político y un sentido de generar conocimiento que se da aquí y en cualquier parte, en cualquier escuela... yo pienso que tiene que marcar la diferencia con el resto... uno gana un sensibilidad frente a las problemáticas.

Así, en los espacios cotidianos de la vida de las organizaciones populares, se afirman o cuestionan, se reproducen o transforman, los $\mathrm{co}$ nocimientos y valores que los sujetos han construido para interpretar y orientar sus prácticas. Las organizaciones se convierten en espa- cios "donde se conversa de otra manera y en los cuales se construye una realidad diferente, opuesta muchas veces, a lo que se vive cotidianamente, por fuera de ella" (Martinic: 1996: 4).

El siguiente testimonio expresa cómo, desde las organizaciones, se generan representaciones contrarias a las predominantes en los ámbitos populares:

\begin{abstract}
Nosotras muchas veces nos agarramos más bien porque queremos que cada ves los niños sean mejor atendidos, esa es una sensibilidad que se gana acá, si, y es que eso de los derechos del niño puede ser que nadie, coja a usted y le recite los derecho de los niños, pero si esta, es la cotidianidad que se vive de otra forma, en el dialogar en el hablar incluso hasta en interponernos con los padres para que ellos no maltraten a sus hijos, una cantidad de cosas que se van ganado poco a poco.
\end{abstract}

También, en las organizaciones se generan esquemas valorativos que reorientan las relaciones de sus integrantes consigo mismos y con los demás; es decir, pautas de interac-

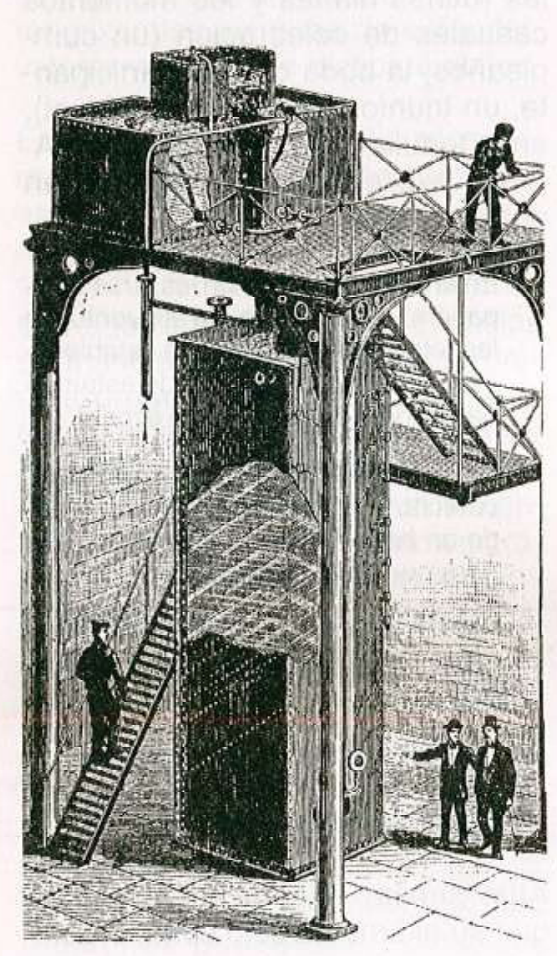

ción, de manejo del poder y de resolución de conflictos, que son interiorizados por sus integrantes para solucionar otras situaciones. Los siguientes testimonios lo confirman:

Igualmente, uno también aprehende a respetar todo eso, respetar lo que piensan los demás, y hacer valer también lo mío y a plantear lo mío, aunque se choque con los demás, cosa que yo tampoco hacía, yo era una persona muy callada, muy en lo mío y ahora no (Sandra, EPI).

Yo pienso, que, en primer lugar, es toda la formación de criterio y de carácter porque, por ejemplo, todavía nos encontramos con gente y me dicen que yo era peliona desde chiquita, pero había algo en el proceso de formación que te hace ser crítico y que te hacia tomar posturas... Eso a mí me marcó muchísimo, o sea, como ese sentido de responsabilidad, el sentido de que... el que tú fuiste subiendo en determinadas cosas pero porque tú le fuiste metiendo el hombro (Mary Sol, FAROL, $\mathrm{CPC}$ ).

Por último, tal como lo plantea Michel de Certeau (1996), desde el quehacer cotidiano de las organizaciones, se producen nuevos, "modos de hacer"; el aprendizaje de dichos saberes prácticos están ligados en las asociaciones populares, al campo de responsabilidades 0 roles que cada uno asume, como a los modos de actuar de los miembros dentro de las mismas:

Entonces aprendí todo lo que fue el manejo de la biblioteca, los criterios y ese tipo de cosas (Mary Sol, CPC).

Nos exigimos muchísimo y también el ser críticos, el utilizar la autocrítica y el evaluarnos, son herramientas propias de Kerigma, el estarnos cuestionando siempre o más que cuestionando es evaluando, eso es Kerigma, un combo de amigos que le apuestan al trabajo bien hecho y digno, de calidad, un trabajo inteligente" (Ricardo, Kerigma).

\section{Los modos cotidianos de aprender}

Nuestra tercera pregunta estaba referida a cómo se logra el cambio de 


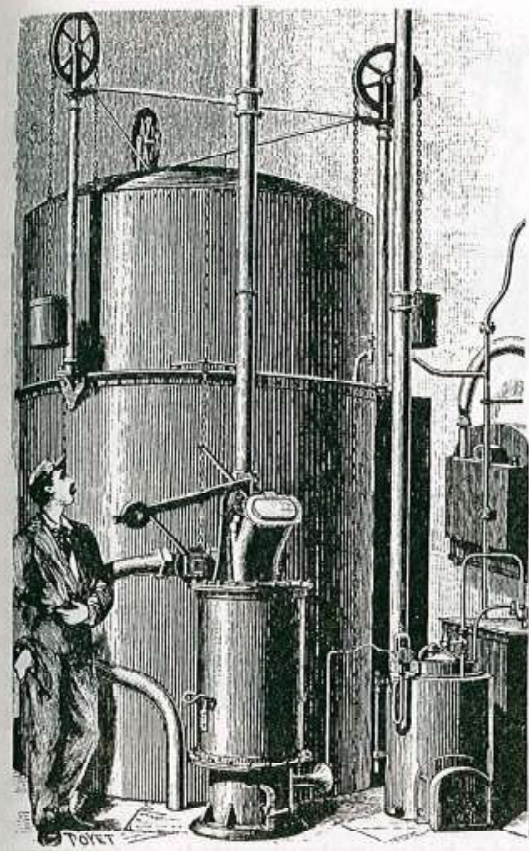

los esquemas interpretativos, valorativos, relacionales y de acción. Ello nos condujo a reconocer las mediaciones, estrategias y procedimientos que posibilitan la formación de los miembros de las organizaciones desde su cotidianidad. Tales mediaciones, principios explícitos o implícitos, así como las maneras de aprehender dentro de las organizaciones, se convierten en las directrices de las prácticas formativas informales.

En el campo de las acciones intencionales, por parte de quienes asumen el rol de formador, tenemos desde el dar un consejo puntual o llamar la atención o insistir sobre los criterios de trabajo de la organización, hasta asumir el acompañamiento de algún miembro "nuevo" de la organización.

Dentro de las tres primeras modalidades, podemos considerar los siguientes testimonios:

Después, me acuerdo mucho, a mí siempre me marcó eso que una vez llegué tarde cinco minutos al taller y María Eugenia no me dejó entrar y me dijo: "Te acostumbras, si tú dices a las tres es a las tres, no es a las tres y cinco"; le dije, pero es que no alcancé a salir, es que tuve lavar algo; "te devuelves y hoy no asistes al taller". Entonces, eso para mí se convirtió en una enseñanza: uno no puede llegar tarde (Mary Sol, CPC).

Me dan la oportunidad de empezar a trabajar con los niños, con la orientación obviamente de ella, ella empezaba como a nivel personal a decirme y a contarme cómo era el niño, cómo evolucionaba, todo el cuento de las áreas y todo este cuento que uno empieza a conocer para poder ya específicamente empezar a trabajar con los niños...

Claudia era muy insistidora, ella era que insista que insista, que hay que hacer esto, que no se qué. Le insisten a uno que uno, uno dice, que le insisten a uno que en verdad es para beneficio propio (Tertulia, EPI).

El acompañamiento es, quizás, uno de las prácticas más significativas del quehacer de los dirigentes de las organizaciones, en cuanto se le concibe como la estrategia principal de formación. Para el CPC, cada persona que hace parte del equipo coordinador ha sido acogido y acompañado de tal manera que, después de llevar un tiempo caminando con el grupo, empieza a acompañar a otros y otras:

Se inició, entonces, un proceso de acompañamiento a este grupo mujeres; abordando inicialmente las problemáticas que más les afectaba y la cual ellas, como habitantes del barrio, podían buscarle una posible solución: los niños (Historia del CPC).

En la medida en que la persona se va sintiendo identificada y comprometida con el grupo al que inicialmente pertenece, se van cambiando las relaciones de contraprestación, por relaciones de gratuidad y solidaridad. Esta estrategia del $\mathrm{CPC}$ tiene su equivalente en Kerigma, donde el voto de confianza y la asignación de responsabilidades a integrantes "nuevos" o en proceso de formación dentro de las organi- zaciones, va posibilitando desarrollar la capacidad de las personas para asumir compromisos mayores.

Otra de las modalidades comunes de aprendizaje son las conversaciones informales, ya sea a partir de las inquietudes de los participantes, de la emergencia de un problema o en los espacios mismos de trabajo compartido; esta interacción dialógica es tal vez, la forma más generalizada de aprender de los demás en la cotidianidad de la organización:

A él yo le aprendí muchísimo, me gustaba comenzar a compartir con él, y comenzarle a decir porqué esto y mis inquietudes, porque yo siempre tuve mis inquietudes y muchas dudas..

...(he aprendido) de esto que es la universidad de la vida, de las mismas personas que coordinan, de los propios compañeros, de los conversatorios que uno tiene informales, yo he aprehendido machísimo, muchísimo, muchísimo (Tertulia EPI).

También se considera una posibilidad de aprendizaje, los conflictos, crisis y problemas que deben, a menudo, enfrentar los integrantes de la organización o ésta en su conjunto:

Por ejemplo, cuando había problemas con los papás, pues a mí nunca me dijeron, este es manual, paso uno: hable con el papá; paso dos; no, sino que a uno siempre le decian, pues hay que demostrar quién es el educador, más allá de decirle pero haga tal cosa. No habia el procedimiento, yo no lo recuerdo realmente, había como el consejo, trato con respeto; pero, por ejemplo, un papá que llegaba como peleón y conflictivo, pues ahí sí le tocaba a uno como aprender. Esa fue una experiencia.

También a uno lo forman a los golpes... acá en la organización, porque, pues también eso hay que decirlo... a veces también en las organizaciones se generan muchos conflictos, tensiones que lo fortalecen a uno, o también mucha gente se ha ido de las mismas organizaciones porque también, no se solucionan esos problemas... (Claudia, EPI). 
Finalmente, algunos testimonios señalan que se aprende en la propia realización de actividades y tareas propias de su rol o campo de acción de la organización:

Pero en la cotidianidad, uno se la anda jugando, se la anda jugando a muchas cosas, a hacer, por ejemplo, lo de los murales es un proceso que cada día se va como que enriqueciendo, que se lee por un lado, que se lee por el otro, el arreglar con el papel kraf (Caudia, EPI).

\section{Confluencia y complementariedad de saberes}

Es necesario aclarar, también, que los participantes de las organizaciones no solamente aprehenden el mundo de la organización, sino que también participan de otros mundos en los que actúan -vida familiar, vida laboral, estudios-, los cuales le dejarán otros esquemas interpretativos, de acción y de relación que confrontará en su diario vivir. La referencia más reiterada es la de los estudios universitarios, motivados por cualificar su propia práctica dentro de las organizaciones.

Es común encontrar, que para los miembros de las organizaciones, lo aprendido en la universidad, complementa los aprendizajes obtenidos en la organización y mejora su práctica dentro de la misma:

Pero como sabemos que de todas formas el hecho de ir a la universidad, y toda la cuestión, hay también ayuda de una cosa con la otra, uno compensa, por decirlo de alguna manera, las dos cosas (Tertulia EPI).

También me marca mucho, mucho, mucho y ahora soy consciente de eso; la formación que yo recibí en la universidad, o sea, cuando estás metido en la universidad no lo valoras, pero la Universidad me aportó mucho porque todo eso que tú traes de lo contestatario, de lo beligerante..., es empezar a madurar tus argumentos: que usted puede ser beligerante tiene que saber argumentar lo que dice; entonces, eso para mí, esas dos cosas han sido complemento (Mary Sol).

Dentro de la historia de Kerigma fue muy importante la formación universitaria de sus miembros, pues la confluencia de diferentes saberes profesionales garantizó el buen nivel de su trabajo artístico (testimonio), y una formación integral de sus miembros, algunos de los cuales son llamados por otras instituciones a prestar sus servicios.

Esta confluencia de aprendizajes en función del trabajo puede ser leída en clave del planteamiento de Schutz (1993), según el cual, la negociación es importante en estos casos, ya que el individuo no es receptor pasivo en la dialéctica marcada por la socialización e interiorización; puede tener cambios de representaciones en la medida que son significativas y tengan un sentido práctico para él.

\section{Reelaboración de necesidades y formación de sujetos}

¿En qué medida las organizaciones reconocen y procesan las necesidades de quienes participan de sus espacios y proyectos?, ¿cómo posibilitan la ampliación de dicho sistema de necesidades?

En primer lugar, hay que puntualizar que para Hugo Zemelman (1993), las necesidades no son carencias propiamente dichas, sino la lectura que hacen individuos y colectivos de dichas carencias, desde su memoria y sus visiones de futuro; en ese sentido, las organizaciones son fuente de nuevas expectativas, aspiraciones, deseos, sueños y utopías, que amplían el sistema de necesidades y posibilitan el despliegue de acciones para resolverlas.

La motivación inicial de los participantes de base de las organizaciones, muchos de los cuales pasan a ser integrantes de las mismas, es

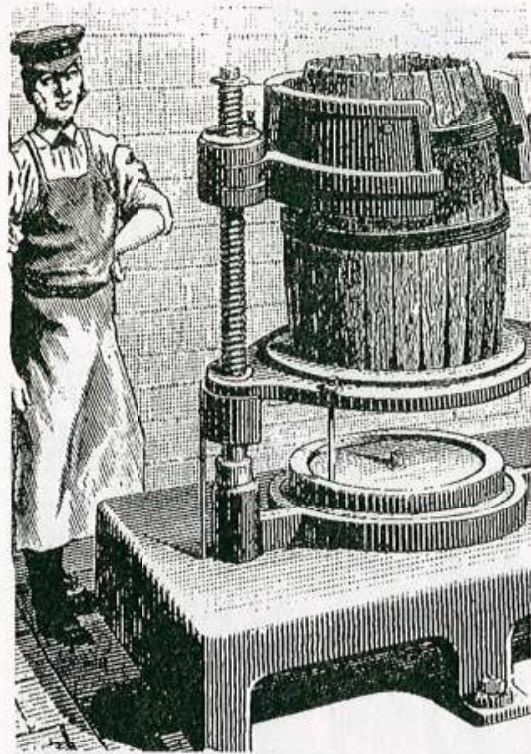

más bien instrumental: satisfacer necesidades individuales básicas (dejar a cuidar sus hijos, acceder al mercado, capacitarse) o simbólicas (de reconocimiento e integración social). Por ejemplo, algunas mujeres que se vinculan FAROL, lo hacen como posibilidad de acceder a recursos como el ahorro que se hace dentro de cada núcleo, que sirve como fondo de solidaridad para calamidades; también se refieren a la oportunidad de "ganarse un mercado", conformado por productos que cada una lleva y cuyas beneficiarias van siendo rotadas, para que todas lo reciban.

Por otro lado, cuando se les preguntó acerca de la incidencia de FASOL en su vida personal, el acento principal estuvo en el establecimiento de vínculos: "conocer gente", "conseguir amigas", "encontrarse con otras mujeres", "tener alguien con quien desahogarse", etc.

En la medida en que las personas se van involucrando con la organización y asumiendo responsabilidades mayores, el horizonte de expectativas personales y sociales se amplía, y también su sistema de necesidades. En el caso de FASOL, la preocupación por mejorar en lo 
personal se proyecta hacia la "comunidad"; la búsqueda de vínculos y amistades individuales pasa a la preocupación por formar parte de redes organizativas.

En las organizaciones populares, como ya se mencionó en el numeral anterior, es común la preocupación de sus integrantes avanzar en sus estudios hasta llegar a los universitarios, en el doble sentido de superación personal y de "ayudar a la organización". Por ello, es que algunos integrantes del CPC, de la EPI y de Kerigma, han estudiado carreras como Trabajo social, Educación Preescolar y otras licenciaturas en el campo pedagógico y artes escénicas.

Como balance global de los procesos formativos vividos (explícitos y cotidianos), quienes han participado durante varios años, reconocen que su identidad personal y social está marcada por la organización. Los valores, conocimientos, modos de hacer y relacionarse aprendidos en ésta, quedan incorporados a su propia personalidad y pueden proyectarlos en cualquier otro espacio social.

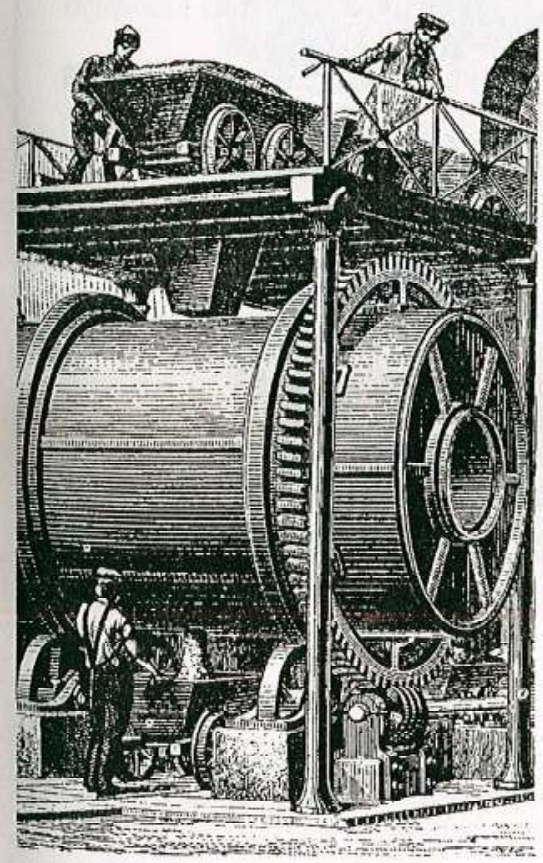

A uno mismo le sirve como mujer, por decir algo, yo me di cuenta que el ser mujer no es sólo la palabra mujer, todo el mundo dice si usted es mujer, y yo eso lo he ganado mucho acá, definitivamente, en la parte pedagógica, o sea yo aquí realmente muchas cosas de lo que soy en este momento.

Quienes se retiran de las organizaciones, es común encontrarlos en otros espacios de trabajo comunitario o proyectando en sus espacios laborales la formación recibida:

Lo que valoré de ese año es que todo lo que yo hice allá era acumulado del Centro o sea mi actuar en ese espacio era el acumulado de lo que yo había hecho con Centro o sea para mí eso fue una de la cosas que más me... porque en últimas el mismo estilo de trabajo, todo ese tipo de cosas yo lo seguía manteniendo (Mary Sol, CPC).

Como conclusión, podemos afirmar que las organizaciones populares, como instituciones socioculturales, forman sujetos capaces de asumirlas y de recrearlas en otros espacios sociales. La "necesidad de organizarse" frente a las circunstancias adversas y los factores que la originan, así como la apropiación de esquemas mentales, valorativos y prácticos para hacerlo, posibilita a quienes se involucran en las organizaciones que se conviertan en actores sociales con visones de futuro y capacidad de generar proyectos y desplegar prácticas que transformen el contexto.

\section{Bibliografía}

Berger, Peter y Luckman, Thomas. La construcción social de la realidad, Buenos Aires, Amorrortu, 2003.

De Certeau, Michel. La invención de lo cotidiano. Las artes de hacer. México, UIAITESO, 1996.

GidDEns, Anthony. La constitución de la sociedad. Bases para una teoría de la estructuración. Buenos Aires, Amorrortu, 1995.

Heller, Agnes. Historia y vida cotidiana, México, Grijalbo, 1980.
Kosık, Karel. Dialéctica de lo concreto, México, Grijalbo, 1979.

MAFFESOLI, Michel. El conocimiento ordinario, México, Fondo de Cultura Económica, 1992.

Magendzo, Salomón y EgAnA, Loreto. La participación social como espacio educativo, Santiago PIIE, 1991.

MARTINIC, Sergio. La construcción dialógica de saberes en contextos de educación popular. Santa Cruz de la Sierra, CEAAL, 1996.

Palma, Diego, La construcción de Prometeo. La educación para la democracia latinoamericana. Santiago, CEAAL -TAREA, 1993.

Regullio, Rosana. Emergencia de las culturas juveniles. Estrategias del desencanto. Bogotá, Norma, 2000.

Sabucedo, J. M. y otros. "Los movimientos sociales: discurso y acción política". En: Revista Universidad de Guadalajara, Guadalajara, Número 11, verano de 1998.

SALete, Roseli. "Los movimientos sociales y la formación de una nueva pedagogía". En El arco iris, Santiago, CEAAL, 1988.

SCHUTZ, Alfred. La construcción significativa del mundo social. Buenos Aires, Paidós, 1993.

Téulez, Gustavo, Pierre Bourdieu. Conceptos básicos y construcción socioeducativa. Bogotá, Universidad Pedagógica Nacional, 2002.

TORRES, Alfonso, "Educación y pedagogía en las experiencias organizativas populares". En La Piragua\# 11, Santiago, CEAAL 1995.

Zemelman, Hugo. "Educación y constitución de sujetos sociales". En: La Piragua \# 7 , Santiago, CEAAL, 1993. 


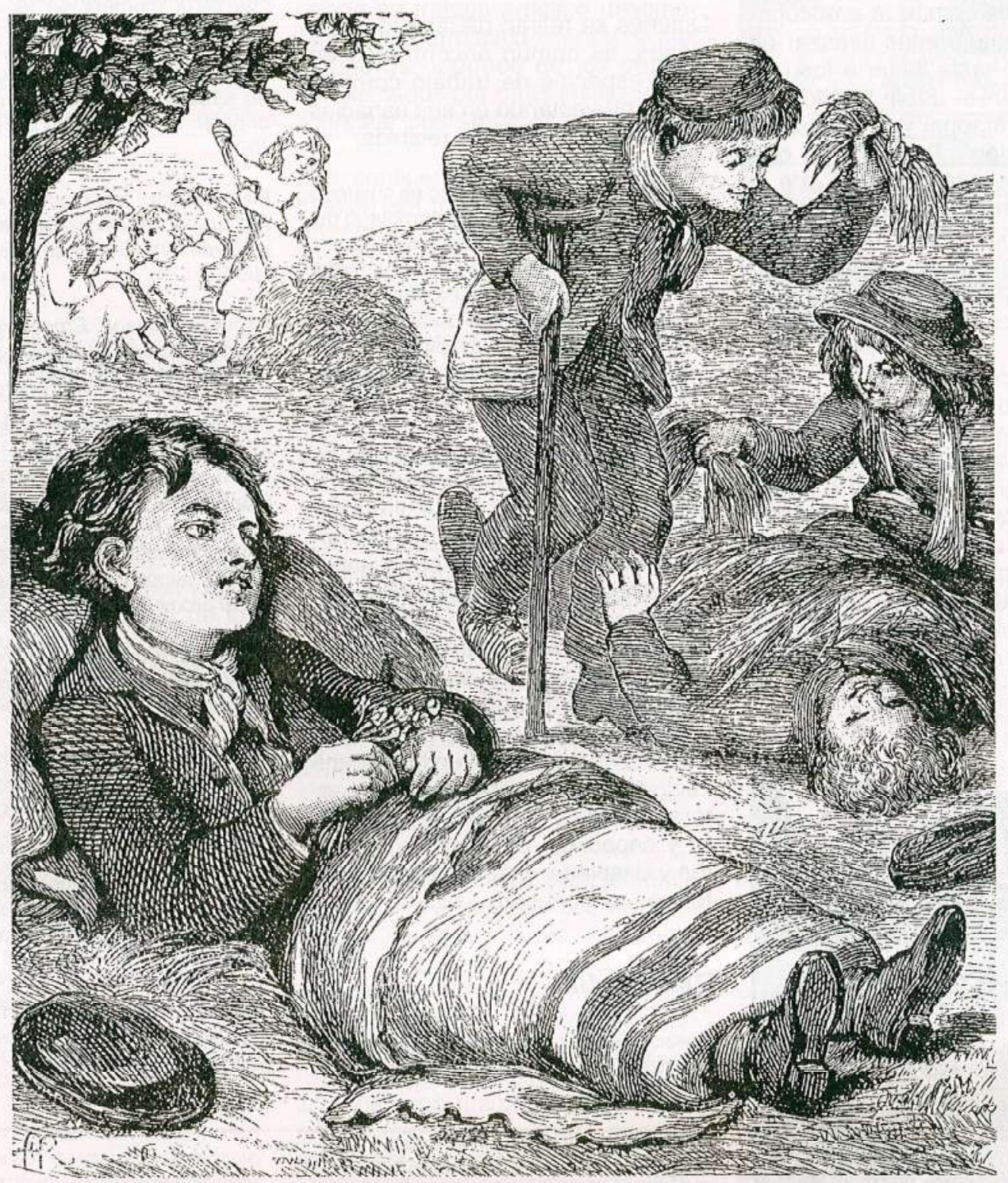

